\title{
Parametric Quadrilateral Meshes for the Design and Optimization of Superconducting Magnets
}

\author{
Martin Aleksa, Stephan Russenschuck, and Christine Völlinger
}

\begin{abstract}
The program package ROXIE has been developed at CERN for the design and optimization of accelerator magnets. The necessity of extremely uniform fields in the superconducting accelerator magnets for $\mathrm{LHC}$ requires very accurate methods of field computation. For this purpose the coupled boundary-element/finite-element technique (BEM-FEM) is used. Quadrilateral higher order finite-element meshes are generated for the discretization of the iron domain (yoke) and stainless steel collars. A new mesh generator using geometrically optimized domain decomposition which was developed at the University of Stuttgart, Germany has been implemented into the ROXIE program providing fully automatic and user friendly mesh generation. The structure of the magnet cross-section can be modeled using parametric objects such as holes of different forms, elliptic, parabolic or hyperbolic arcs, notches, slots, .... For sensitivity analysis and parametric studies, point based morphing algorithms are applied to guarantee smooth adaptation of the mesh to geometry changes. For modeling three-dimensional iron structures, the 2-D meshes can be extruded into the third dimension. This paper discusses the use of the mesh generator with examples of the computation of the LHC dipoles.
\end{abstract}

Index Terms-Mesh extrusion, mesh generator, morphing algorithm, quadrilateral finite elements.

\section{INTRODUCTION}

$\mathbf{T}$ HE DESIGN of the superconducting LHC magnets is dominated by the requirement of an extremely uniform field, which is mainly defined by the layout of the superconducting coils. The ROXIE [1] program package was therefore developed at CERN for the design and optimization of the LHC magnets and includes the method of coupled boundary/finite-elements (BEM-FEM), which was developed at the University of Stuttgart, Germany [2], and which is specially suited for the calculation of 3-D effects in the magnets. The advantage of this method is that the coils do not need to be represented in the finite-element mesh and can therefore be modeled with the required accuracy.

The iron yoke with its nonlinear magnetization behavior is modeled with finite elements (FEM) and couples to the boundary element domain (BEM) on the common boundary between iron and air. Simplex elements (triangles in 2-D, tetrahedra in 3-D) have the disadvantage that curved shapes can only be modeled by polygonal approximations. The advantage of a higher order approximation of the potentials may be lost due to a rather rough geometric approximation. An alternative are the isoparametric quadrilateral elements which have curved sides. Numerically unfavorable prisms can be avoided when

Manuscript received September 24, 2001.

The authors are with CERN, 1211 Geneva 23, Switzerland.

Publisher Item Identifier S 1051-8223(02)04207-0. the geometry discretized with quadrilateral elements is simply extruded into the third dimension.

A new mesh generator based on geometrical domain decomposition, which was also developed at the University of Stuttgart [3] has now been implemented in the ROXIE program package. The following extensions have been added.

1) Extension of the method to eight-noded (higher order) quadrilateral elements.

2) Parametric input for the definition of design variables for the mathematical optimization.

3) Implementation of design features for the definition of material boundaries.

4) Modular magnet-geometry input by means of the GNU $\mathrm{m} 4$ macro language [4].

5) A morphing algorithm for optimization and sensitivity studies which avoids re-meshing and changing of mesh topologies during optimization.

6) The extrusion of 2-D meshes into the third dimension to permit full 3-D computation of nonlinear iron structures.

The method is now available in the ROXIE 8.2 version. This report gives a short description of the method and presents the field quality calculations for the prototype and pre-series LHC dipole magnets.

\section{The Mesh Generator}

The quadrilateral mesh generator implemented in ROXIE 8.2 relies on the method of geometrical domain decomposition [3].

In a first step the input geometry [see Fig. 1(a)] is decomposed into single connected areas, i.e., holes are eliminated [see Fig. 1(b)]. Fig. 1(c) shows the result of the geometric domain decomposition. The exact position of the cuts is optimized by minimizing a function that depends on the ratios $l^{2} / A, l / U$, and the angles of the old and the newly created areas, where $l$ is the length of the cut, $A$ stands for the cut area, and $U$ is the circumference of the cut area. This decomposition is continued until the remaining areas are regarded as "simple ${ }^{1}$." These areas are then "filled" with quadrilaterals using a modified paving strategy [5]. In this approach an area is "filled" from outside to the inside by adding full rows of rectangles. The finite-element mesh obtained after "filling" the decomposed geometry is presented in Fig. 1(d).

In some works (see e.g., [6]) the geometrical domain decomposition has been carried on until the remaining areas only consisted of six nodes. ${ }^{2}$ For the rather fine meshes that have to be

\footnotetext{
${ }^{1}$ Areas are regarded as "simple" when they are "similar" to triangles or rectangles, see Fig. 1(c) and [3].

${ }^{2}$ Such a domain was finally divided into two four-noded elements.
} 
(a)

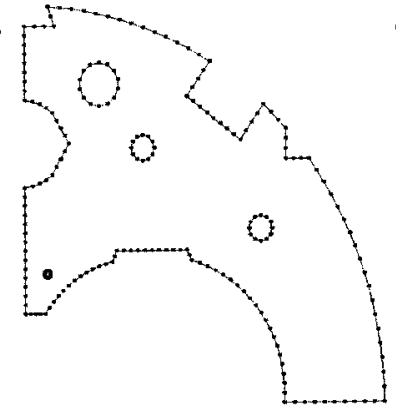

(c)

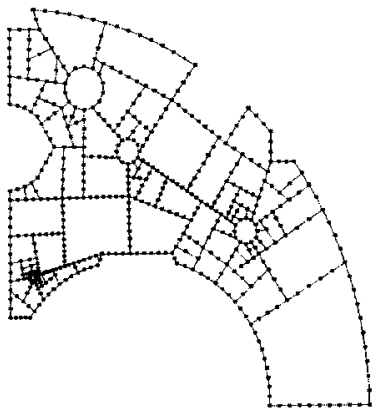

(b)

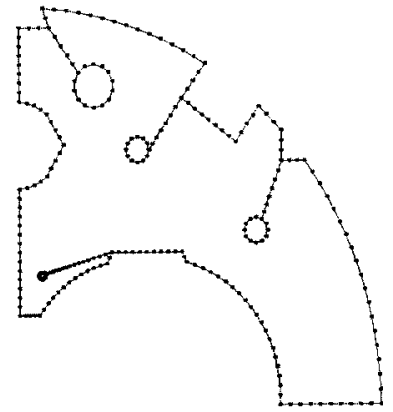

(d)

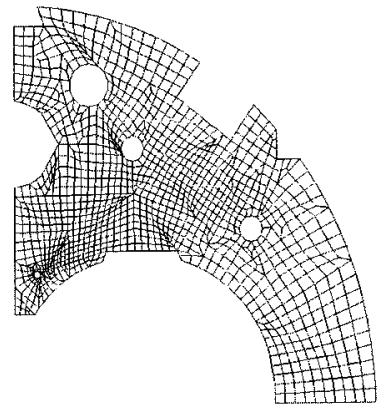

Fig. 1. The different steps of the quadrilateral mesh generation using geometrical domain decomposition. Plot (a) shows the input contour polygon, (b) the geometry after the topology decomposition, (c) the geometrically decomposed geometry and (d) the finite-element mesh before smoothing.

used for magnet optimization, this strategy would be too time consuming. The time consumption of the decomposition of an area with $m$ contour points grows with the order of $m^{3}$ [3] if an area gets cut into half each time, or is proportional to $m^{4}$ if only small pieces of four nodes are cut away (worst case). This shows the importance of minimizing $l^{2} / A$ during the decomposition. In contrast to that, the time consumption for filling "simple" areas with quadrilateral meshes grows with the order of $m^{2}$ [3], demonstrating the superiority of the applied method for complicated geometries (i.e., large $m$ ).

Finally, smoothing algorithms are applied. A linear combination of Laplace-smoothing, edge-smoothing and angle-smoothing is used to enlarge small angles, to reduce large angles, and to increase short distances between mesh points by leaving the topology unchanged.

Since the mesh generator described above produces finite elements with four nodes, the middle points of the finite-element edges are inserted according to their position on arcs, circles, etc. during post processing and eight-noded (higher order) quadrilateral elements are produced which are more favorable for three dimensional field computation after being extruded to 20-noded hexahedras.

\section{The Modeling of the Magnets}

The new quadrilateral mesh generator is invoked by the keyword "HyperMesh;" at the beginning of the iron file. ${ }^{3}$ For a more detailed description of the input syntax see [7].

The geometry has to be described with key-points which are connected by lines. In order to facilitate the geometry input line macros (using the "HyperLine" command) are supplied that

\footnotetext{
${ }^{3}$ The iron file serves as an input file to ROXIE, providing the data describing the iron geometry (e.g., the iron yoke and the insert)
}

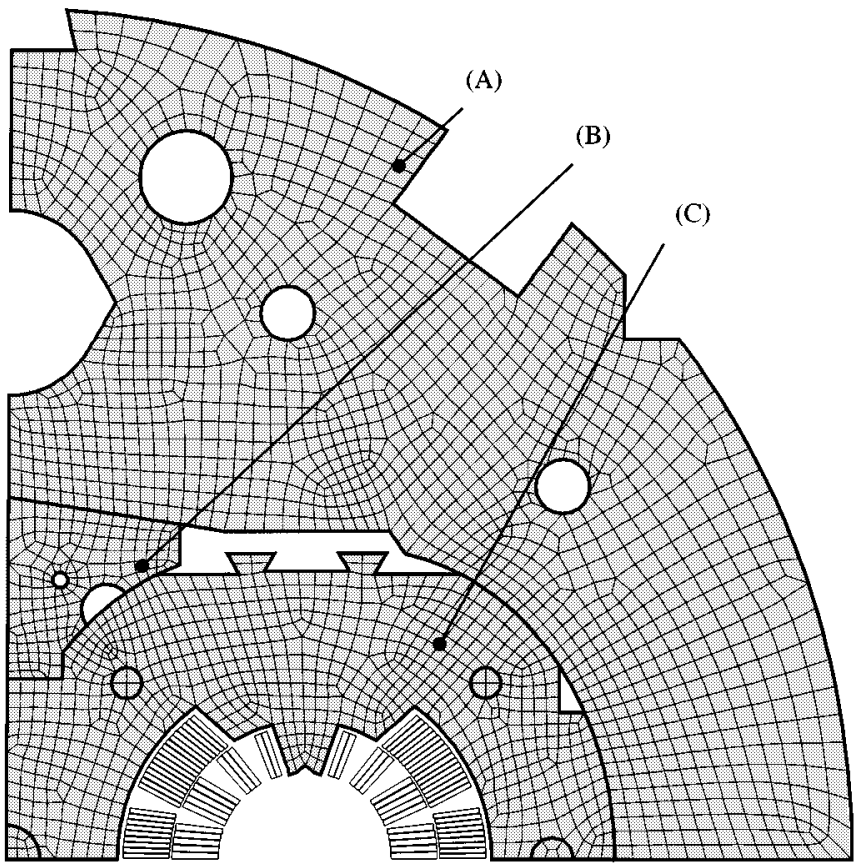

Fig. 2. Finite element mesh of the LHC main dipole iron yoke (A), insert (B), and stainless steel collar, and (C) created with the new mesh generator.

include all conic sections, circular, rectangular and elliptic holes as well as different design features such as notches, bars and corners. These macros are easy to use and only a few meaningful parameters have to be supplied by the user. They allow a very precise modeling of the exact magnet geometry.

In any case, the parametric definition of key-points and lines can be done using FORTRAN-type syntax that allows for all the standard functions and dependences. The symbols used for the definition of key-points and lines can be addressed as design variables for the optimization process.

Areas have to be defined with the "HyperArea" command by listing all the lines enclosing them. One entirely new concept for the mesh generation of ROXIE are holes within area elements. They have to be declared using the "HyperHole0f" command.

The number of subdivisions for the contour lines can be set using the "Lmesh" command. In this way the density of the mesh can easily be adjusted to the geometry.

Using the GNU $\mathrm{m} 4$ macro package, magnetically relevant parts of the magnet (e.g., yoke, collar, inserts, cryostat, beam-screen etc.) can be modeled in different iron files and can then be linked with the "include" command. In such a way it is easy to switch "on" and "off" certain parts of the geometry. In the same way, it is possible to re-use parametric models for different magnet variants.

\section{RESUltS OF THE LHC MAIN DipOLE}

As an example for the abilities of the mesh generator the LHC main dipole magnet was recalculated for the geometry of the iron yoke used in the pre-series LHC dipoles. This model takes into considerations all the changes to the yoke and insert geometry which have been incorporated since the end of 1998.

One big advantage of the new mesh generator is the possibility to adapt the density of the mesh to the gradients of the 


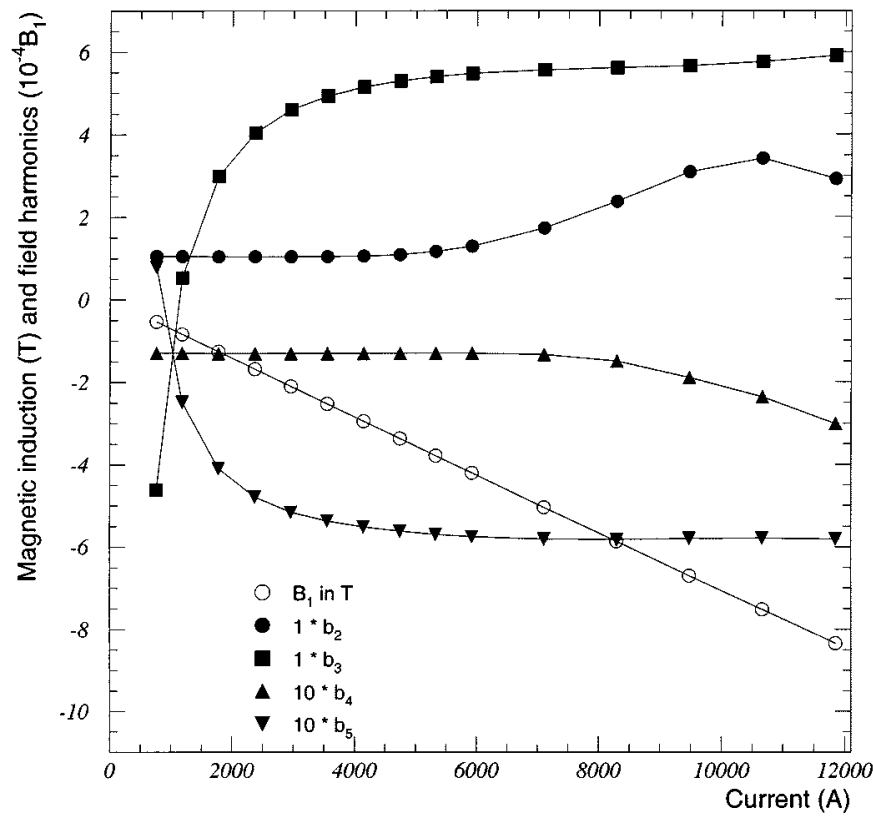

Fig. 3. Load line of the LHC main dipole including the effect of persistent currents in the superconducting filaments [8]. The main field component $B_{1}$ and the relative field components $b_{2} \cdots b_{5}$ are plotted (up-ramp cycle).

field. Fig. 2 shows the meshed iron yoke, the insert, and the collar (stainless steel) for the BEM-FEM field calculation. The mesh density can easily be changed by modifying the subdivisions on the contour lines.

The calculations were performed for a number of current levels between injection and nominal field level (up-ramp cycle) including the calculation of persistent currents in the superconducting filaments as described in [8]. The relative permeability $\mu_{r}$ of the stainless steel collar is assumed to be 1.0025. Fig. 3 shows the relative multipole errors $b_{n}$ at a $17 \mathrm{~mm}$ reference radius in units of $10^{-4} \cdot B_{1}$ and the main field component $B_{1}$ in Tesla. The nonlinear behavior of the relative field components $b_{3}$ and $b_{5}$ for low fields is due to the effect of persistent currents.

Fig. 4 shows the $z$-component of the vector potential $A$ and Fig. 5 shows the modulus of the magnetic induction $|B|$. Both plots are at nominal field level $\left(I=I_{0}=11850 \mathrm{~A}\right.$, main field component $B_{1}=-8.34 \mathrm{~T}$ ).

\section{PARAMETERIZATION FOR OPTIMIZATION AND SENSITIVITY STUDIES}

In order to make the new mesh generator applicable for optimization and sensitivity studies, the finite element meshes have to be "parameterized." If design variables that determine the iron geometry change, the mesh has to be adapted to the new geometry. Ideally, small changes of the geometry should cause only small shifts of the mesh but keep the mesh topology unchanged.

\section{A. Re-Meshing}

One easy way to realize mesh changes with updated iron geometries is to calculate the new contour lines and to re-mesh each time. Investigations of the evolution of the mesh during a parameter study and its impact on the field harmonics showed

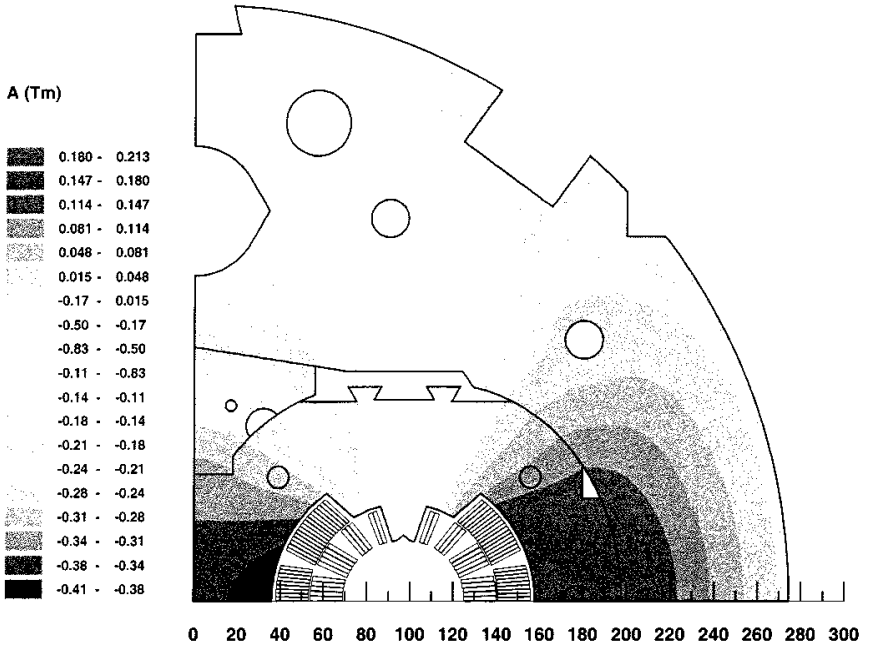

Fig. 4. Vector potential $A$ ( $z$-component) in the iron yoke and the stainless steel collar at nominal field level.

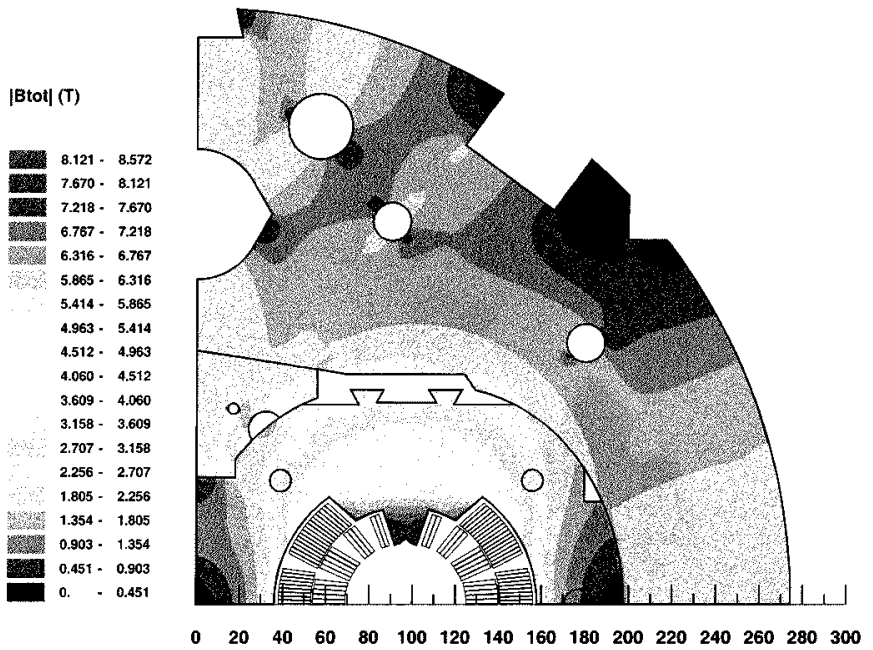

Fig. 5. Modulus of the magnetic induction $|B|$ of the main dipole including the stainless steel collar at nominal field level.

that this solution is only little satisfying since changes in the mesh topology and hence discontinuities of the field harmonics have been observed.

Such discontinuities obviously have a strong impact on the convergence of optimization algorithms and decrease strongly their efficiency especially if gradient based optimization strategies are used. Even though the absolute error introduced by mesh-topology changes is rather small $(\sim 1 \%)$, the discontinuity of the relative field components and hence the discontinuity of the objective function will have a strong impact on the convergence of optimization algorithms since they very often rely on the monotony of the objective function.

The most elegant way out of this dilemma is the application of a morphing algorithm and hence keeping the mesh topology unchanged.

\section{B. Point Based Morphing}

Morphing is a technique known from computer graphics (see e.g., [9]) and is usually used to transform pictures. 

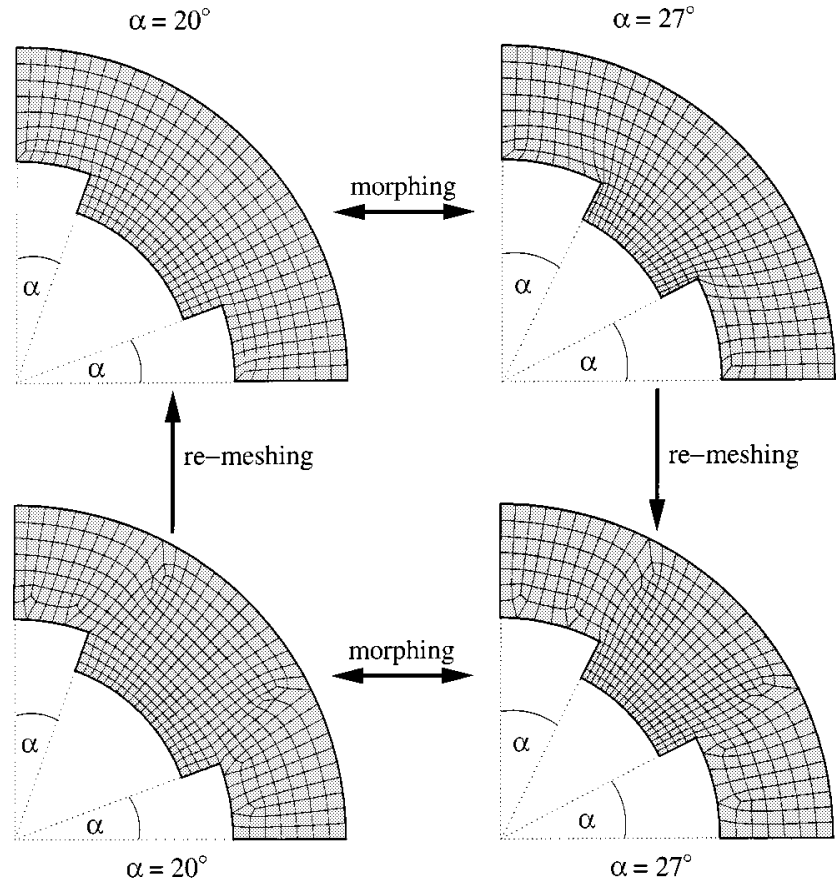

Fig. 6. Demonstration of point based morphing with a simple iron yoke for a quadrupole corrector magnet.

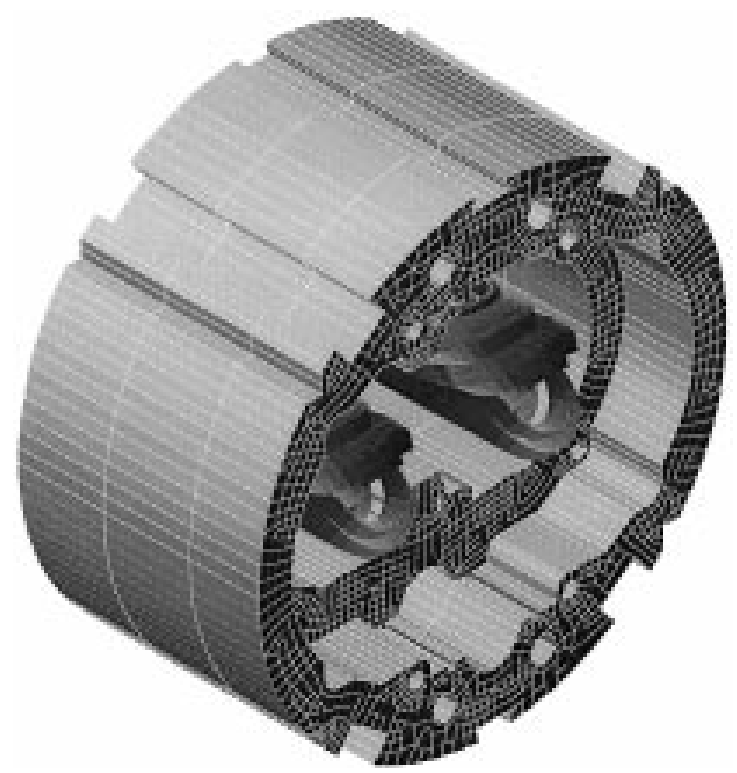

Fig. 7. Three-dimensional mesh obtained by extruding the 2-D mesh into the third dimension.

Point based morphing uses some given translation vectors $\vec{v}$ defined explicitly at some point locations $p_{i}, i=1, \ldots, n$ (in our case the points of the contour). Then the translation vector $\vec{v}$ of any given point $p$ can be calculated as

$$
\vec{v}(p)=\frac{\sum_{i=1}^{n} \frac{\vec{v}\left(p_{i}\right) \cdot w_{i}}{\overline{p p_{i}}}}{\sum_{i=1}^{n} \frac{w_{i}}{\overline{p p_{i}}}}
$$

where $w_{i}$ are weighting factors (in our case $w_{i}=1$ ) and $\overline{p p_{i}}$ denotes the distance between points $p$ and $p_{i}$.
Applying this algorithm to the finite element meshes produces smooth transformations and hence conserves the mesh topology. Fig. 6 demonstrates the application of point based morphing to the mesh of a simple iron yoke. In the upper two figures the mesh has been produced for $\alpha=20^{\circ}$ whereas in the two figures below the mesh has been produced for $\alpha=27^{\circ}$. Note the different mesh topologies in the upper and lower figures.

\section{EXTRUSION PARALLEL TO THE $z$-AXIS}

The field computation program ROXIE is also used to design the coil ends of accelerator magnets. For this purpose a 3-D modeling of the iron structure is necessary. In order to yield three-dimensional meshes the 2-D quadrilateral meshes can be extruded parallel to the $z$-axis by using the "HyperExtrude" command. Each area of the 2-D mesh can be extruded to different lengths along the $z$-axis. Fig. 7 shows an example of a 3-D mesh, modeling the two-layered iron yoke in the end region of the LHC main dipole magnet. For better visibility the inner part of the iron yoke has been elongated by $10 \mathrm{~cm}$ with respect to the LHC dipole design.

\section{CONCLUSION}

A new mesh generator using geometrical domain decomposition has been implemented into the CERN field computation program ROXIE. It produces 8 noded (higher order) quadrilateral finite elements which can exactly model the material boundaries and are advantageous for three-dimensional calculations. Many design features useful for the modeling of superconducting magnets have been defined as macros. A point based morphing algorithm has been implemented in order to transform the finite element mesh smoothly and without topology changes during optimization. For 3-D calculations an extrusion of the 2-D mesh parallel to the $z$-axis has been implemented. The results of the field-error calculations for the LHC main bending dipole have been presented as an example.

\section{REFERENCES}

[1] S. Russenschuck et al., "ROXIE: Routine for the optimization of magnet $\mathrm{x}$-sections, inverse field calculation and coil end design,", CERN 99-01, 1999.

[2] S. Kurz and S. Russenschuck, "The application of the BEM-FEM coupling method for the accurate calculation of fields in superconducting magnets," Electrical Engineering, 1999.

[3] G. D. Nowottny, Netzerzeugung durch Gebietszerlegung und duale Graphenmethode. Aachen: Shaker Verlag, 1999.

[4] GNU m4, version 1.4.. [Online]. Available: http://www.array.ca/products/ipe-1.2/doc/gnu/m4/m4_1.html.

[5] T. D. Blacker et al., Int. J. Numer. Method. Eng., vol. 32, pp. 811-847, 1991.

[6] S. W. Chae and J. H. Jeong, "Unstructured surface meshing using operators," in Proc. 6th Int. Meshing Roundtable, 1997, pp. 281-291.

[7] M. Aleksa and S. Russenschuck, "A new quadrilateral mesh generator for LHC magnet optimization,", LHC-PROJECT-NOTE-233, 2000.

[8] M. Aleksa, C. Völlinger, and S. Russenschuck, "Magnetic field calculations including the impact of persistent currents in superconducting filaments," IEEE Trans. Magn., 2001, submitted for publication.

[9] T. Beier et al., "Feature-based image metamorphosis," Computer Graphics, vol. 26, no. 2, 1992. 